\title{
On-line Handwritten Arabic Character Recognition using Artificial Neural Network
}

\author{
Khaoula ADDAKIRI \\ Department of Mathematics and Computer \\ Science, \\ Université Hassan $1^{\text {er }}$, FSTS, LABO LITEN \\ Settat, Morocco
}

\author{
Mohamed BAHAJ \\ Department of Mathematics and Computer \\ Science, \\ Université Hassan $1^{\text {er }}$, FSTS, LABO LITEN \\ Settat, Morocco
}

\begin{abstract}
In this paper, an efficient approach for the recognition of online Arabic handwritten characters is presented. The method employed involves three phases: First, pre-processing in which the original image is transformed into a binary image .Second, training neural networks with feed-forward back propagation algorithm .Finally, the recognition of the character through the use of Neural Network techniques. The proposed approach is tested on 1400 different characters written by ten users. Each user wrote 28 Arabic characters five times in order to get different writing variations. Experiment results showed the effectiveness of our approach for recognizing handwritten Arabic characters.
\end{abstract}

\section{Keywords}

Pattern Recognition, Neural Networks, Arabic Handwritten.

\section{INTRODUCTION}

In the recent decades, pattern recognition community has undergone a great deal of research in area of handwritten character recognition. Many papers about applications of handwritten recognition to automatic reading of postal address, bank checks and forms (coupons, invoices, document etc.) have been published [1-5]

Considerable work has been undertaken in the area of Arabic character recognition but with limited success, this is due to the complexity of Arabic characters and lack of public Arabic handwriting databases. Arabic alphabet consists of 28 basic characters. Some characters may have different shapes depending on their position within a word (beginning, middle, end) and different size (height and width).In addition, sixteen of the Arabic characters have a single dot, or double, or triple dots or a zigzag (hamza) which are used to distinguish between characters having identical main parts [6].

The aim of this paper is to develop a method that recognize on-line Arabic handwritten characters, Each character has different features that distinguish it from other characters, especially the character that contain sharp edges like: ج, ح, $, \dot{\tau}, \varepsilon$ $\dot{\varepsilon}$. Our approach is Artificial neural network based on Multilayer perceptron (MLP) used to recognize Arabic handwritten characters. The proposed system consists of three main phases. First, while a user writes a character on a special window on the screen, the $(\mathrm{x}, \mathrm{y})$ coordinates of the pixels forming the character are captured then stored in an array. Second, a bounding box is drawn around the character and then features that give structural information of the character are extracted. Then, the character can be classified in order to recognize by using gradient descent learning method for feed forward neural network. The Experiment results showed the effectiveness of our approach for recognizing handwritten Arabic characters.

The rest of the paper is organized as follows. In Section 2, an overview of the Arabic language is presented. Section 3 presents some related work. An overview of the proposed approach including the feature extraction algorithms are discussed in Section 4. Experimental results and discussions are presented in Section 5. Conclusions are presented in Section 6.

\section{OVERVIEW OF THE ARABIC LANGUAGE}

Arabic language is universal and it is a formal language for 25 countries and written by more than 250 million people. Arabic presents one of the major worldwide document sources [6]. By its nature, Arabic text is written cursively, which makes its recognition more difficult than that of printed Latin or Chinese text. On the other hand, Arabic writing, in a similar way to English, uses letters. The Arabic alphabet consists of 28 letters, and text is written from right to left in a cursive way. Each Arabic letter has either two or four shapes depending on its possible position in the text, including start, middle, end, or alone (7-8).

For example, the letter ghayn has the following shapes: start (a), middle (b), end (c), and alone (d), see Fig.1

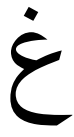

(d)

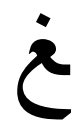

(c)

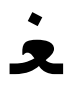

(b)

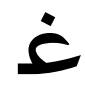

(a)
Fig. 1: Different forms of "GHAYN" character.

The details of the letter shapes are illustrated in Table 1, and evidently this has brought more difficulty for automatic recognition of Arabic texts.

The Arabic alphabet is represented numerically by a standard communication interchange code approved by the Arab Standard and Metrology Organization (ASMO) [9]. Similar to the American Standard Code for Information Interchange (ASCII), each character in the ASMO code is represented by one byte. An English letter has two possible shapes. One is termed "Capital shape" while the second is called "Small shape". The ASCII code provides separate representations for both Capital and Small shapes. The Arabic letter has just one representation in the ASMO table. This is not to say that an Arabic letter has only one shape. On the contrary, most of 
Arabic letter might have up to four different shapes, depending on its relative position in the text.

Table 1: Arabic letter shapes

\begin{tabular}{|c|c|c|c|c|}
\hline Letter & Single & Ending & Middle & Beginning \\
\hline Alef & 1 & 1 & 1 & 1 \\
\hline Baa & ب & ب & ب ب & بـ \\
\hline Taa & 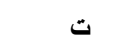 & 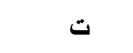 & 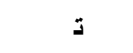 & ت \\
\hline Thaa & 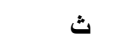 & 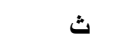 & $\dot{\Xi}$ & $\dot{\jmath}$ \\
\hline Jeem & ج & ج & ج & ج \\
\hline Haа & $\tau$ & ج & $\boldsymbol{~}$ & 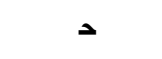 \\
\hline Khaa & $\dot{\tau}$ & غ & $\dot{\Delta}$ & $\dot{~}$ \\
\hline Dal & $د$ & د & د & $د$ \\
\hline Thal & $\dot{j}$ & $\dot{j}$ & j & $\dot{j}$ \\
\hline Raa & J & J & J & J \\
\hline Zai & j & j & j & j \\
\hline Seen & س س & س س س & سد & سد \\
\hline Sheen & ش & ش ش & 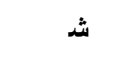 & ش ش \\
\hline Sadd & ص & ص & ص & ص \\
\hline Dadd & ض & ض ض & ض & ض \\
\hline Tah & b & $b$ & $b$ & $b$ \\
\hline Thah & ط & b & b & ط \\
\hline Ayn & $\varepsilon$ & $\varepsilon$ & 2 & $=$ \\
\hline Ghyn & $\dot{\varepsilon}$ & $\dot{\varepsilon}$ & $\dot{z}$ & $\dot{\varepsilon}$ \\
\hline Faa & ف & ف & ف & $\dot{9}$ \\
\hline Qaf & ق & ق & ق & ق \\
\hline Kaf & كs & s & $s$ & $s$ \\
\hline Lam & J & J & $J$ & $J$ \\
\hline Meem & P & b & ـ & هـ \\
\hline Noon & ن & ن ن & $\dot{j}$ & $\dot{j}$ \\
\hline На & 。 & d & $t$ & $\dot{j}$ \\
\hline Waw & 9 & 9 & 9 & 9 \\
\hline Yaa & ي & ي & ي & $\Rightarrow$ \\
\hline
\end{tabular}

\section{RELATED WORK}

For the past few decades, intensive research has been done to solve the problem of Arabic character recognition. The main objective of this section is to present the state of Arabic character recognition research throughout the last two decades. The previously related work can be described as:

El-Sheikh et al. [10] [11] presented two algorithms to recognize cursive words and Arabic handwritten characters. Four different sets of character shapes have been independently considered (initial, medial, final, and isolated).

El-Khaly et al. [12] presented an algorithm for the machine recognition of optically captured Arabic characters and their isolation from the printed text. Moment-invariant descriptors are investigated for the purpose of recognition of individual characters.

El-Wakil et al. [13] proposed a method for the recognition of isolated handwritten Arabic characters drawn on a graphic tablet. Two types of features are extracted from the characters. Features that are independent of the writer style are represented as a list of integer values, while those that are subjected to more variations are represented using a Freemanlike chain code.

Sabri Mohmoud [14] has used Fourier and contour analysis for the recognition of Arabic characters. The features of an input character are compared to the models' features using a distance measure. The model with the minimum distance is taken as the class representing the character.

Cheung et al. [15] presented an Arabic OCR system, which uses a recognition-based segmentation technique to surmount the classical segmentation problems. There is also a feedback loop to control the combination of character fragments for recognition.

Kharma et al. [16] proposed the use of mapping for the recognition of on-line handwritten characters. This mapping produces the same output pattern regardless of the orientation, position, and size of the input pattern.

Mezghani et al. [17] investigated a method for on-line Arabic characters recognition. This method is based on the use of Kohonen maps and their corresponding confusion matrices which serve to prune them of error-causing nodes, and to combine them consequently.

Alkhateeb et al. [18] described an offline Arabic cursive text using Hidden Markov Models and re-ranking. Several experiments are performed using the IFN/ENIT benchmark database.

Vaseghi et al. [19] presented a holistic system for the recognition of handwritten Farsi/Arabic word using right-left discrete Hidden Markov Models (HMM) and kohonen selforganizing vector quantization for reading names in postal addresses. 
Al-Taani et al. [20] proposed an efficient approach for the recognition of Arabic character by using structural features and decision tree learning techniques.

\section{PROPOSED TECHNIQUE}

In this paper, we proposed an on-line recognition system for the handwritten Arabic using neural networks. The whole system contains three stages in terms of preprocessing, feature extraction, and classification in the following sections. The block diagram of the proposed handwritten Arabic recognition system is shown in Fig. 2. As shown in Fig. 2, once a sample image is acquired, pre-processing is required to standardize the signal for better performance in the following stages. Afterwards, features are extracted. These extracted features will then provide as input patterns to neural networks system. Once the neural network system has been trained for these input patterns, it will be able to classify them. Relevant techniques are discussed in details as follows.

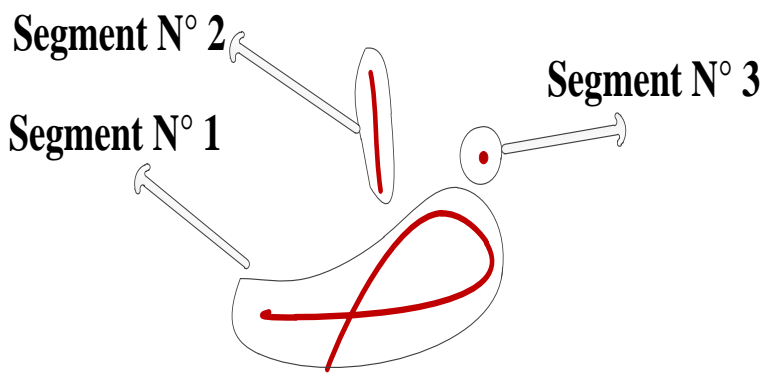

Fig. 3: Three-segment Letter (THAH Letter)

\subsubsection{Cross-Points (Loop)}

Another feature which is useful is whether the written letter contains a loop or not. Nine of the Arabic letters contain loops Fig. 4

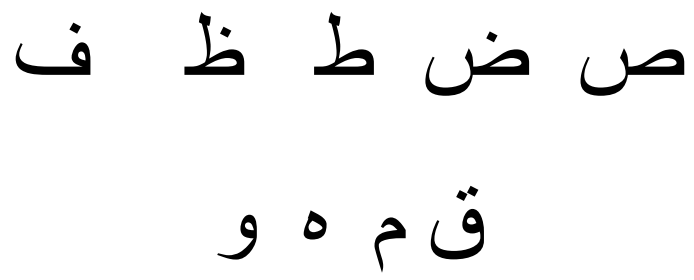

Fig. 4: Arabic letters that have a loop.

Test data 4.2.3 Sharp Edges (ShE)

The Sharp Edge (ShE) detection is one of the most difficult features to be extracted. Sharp edge is similar to 20-40 degree angle. To illustrate this feature see Fig. 5 that shows some letters that have sharp edges. In Figure 5(a) the letter "AYN" is a y-direction ShE type while in Fig. 5(b) the letter "SAAD" is an $\mathrm{x}$-direction ShE type. The y-direction sharp edge is detected during the movement of the pen from upward to downward, and the x-direction is detected when a sharp turning point exists with the movement from right to Left.
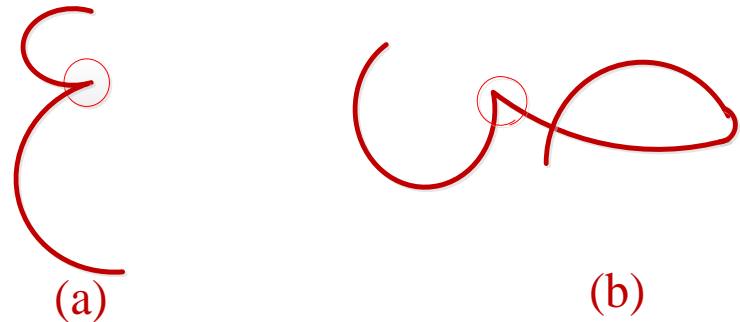
information. In preprocessing phase it is being normalized and removing all redundancy errors from the character image and sends it to next stage of feature extraction.

\subsection{Feature Extraction}

\subsubsection{Number of Segments (NS)}

Number of Segments (NS) is the most important feature used in this work. By segment we mean the separate letter component that must be written without lifting the pen. Fig. 3 shows the Letter "THAH" that has three segments. 
shows the block diagram of the MLP neural network. A three layer MLP with enough number of nodes in.

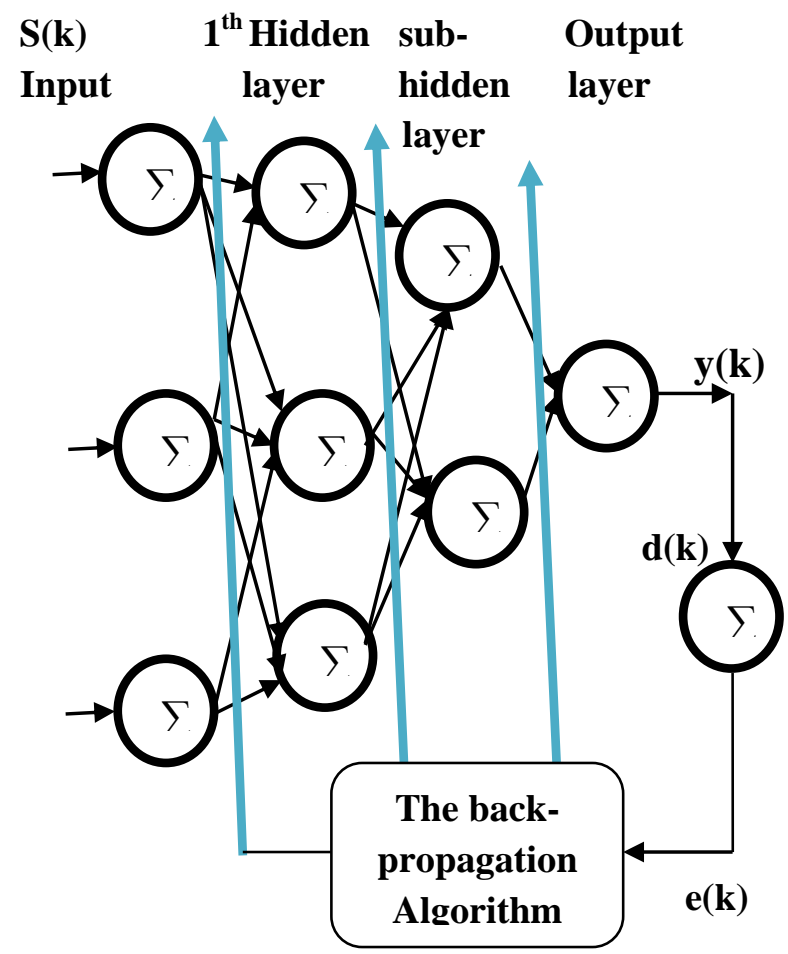

Fig.6. Block diagram of the MLP neural network

The hidden layer is able of approximating any arbitrary nonlinear mapping between the input and output space. The input pattern $\mathrm{s}(\mathrm{k})$ and its corresponding desired pattern $\mathrm{d}(\mathrm{k})$ are applied to the network.

The MLP produces an output $\mathrm{y}(\mathrm{k})$ is obtained as:

$$
y(k)=f\left(\sum_{J=0}^{n-1}\left(s_{j} w_{j}\right)+b\right)
$$

Where, $b$ is the bias and $w_{j}$ the weight matrix associated with input line $\mathrm{j}$. Which is then compared with the desired pattern $\mathrm{d}$ (k) producing an error signal e (k) is obtained as:

$$
e(k)=d(k)-y(k)
$$

The error signal is propagated back to the hidden layers. The weights and thresholds associated with the network are updated using the back propagation (BP) algorithm [22]. This step is repeated till the mean square error (MSE) of the network approaches a minimum value. The MSE at $\mathrm{k}^{\text {th }}$ time index may be defined as:

$$
E(k)=\sqrt{\frac{1}{n}} \sum_{j=1}^{n}\left[e_{i}(t)^{2}\right]
$$

\section{RESULTS AND DISCUSSIONS}

The features (Number of Segments, Cross-Points and Sharp Edges) extracted in the previous phase are used in the classification phase. Neural networks method is used to recognize the Arabic letters: First of all, training of system is done by using different features and then system is tested. The data set was divided into two parts. The first is used for training the system and the second part was for testing purpose.

Ten users tested the proposed method. Each user wrote the 28 letters of the Arabic five times. Every letter was written fifty times and the test set size used in the experiments is 1400

\begin{tabular}{|c|c|c|c|}
\hline Character & Percentage & Character & Percentage \\
\hline$T$ & $94 \%$ & ض ض & $80 \%$ \\
\hline ب & $90 \%$ & b & $85 \%$ \\
\hline 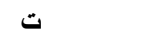 & $\mathbf{9 0 \%}$ & b & $80 \%$ \\
\hline 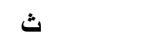 & $60 \%$ & $\varepsilon$ & $60 \%$ \\
\hline ج & $50 \%$ & $\dot{\varepsilon}$ & $90 \%$ \\
\hline$\tau$ & $70 \%$ & ف & $85 \%$ \\
\hline$\dot{\tau}$ & $\mathbf{9 0 \%}$ & ق ق & $83 \%$ \\
\hline$د$ & $\mathbf{9 0 \%}$ & s & $90 \%$ \\
\hline$\dot{j}$ & $88 \%$ & J & $95 \%$ \\
\hline J & $88 \%$ & م & $85 \%$ \\
\hline j & $85 \%$ & ن & $93 \%$ \\
\hline س & $85 \%$ & 。 & $80 \%$ \\
\hline ش ش & $85 \%$ & g & $84 \%$ \\
\hline ص & $80 \%$ & ي & $85 \%$ \\
\hline
\end{tabular}
letters. Experimental results are presented in Table 2.

Table 2: Recognition rates for Arabic letters in the system.

Experimental results showed that the proposed method gave a recognition rate of about $83 \%$ for all letters, and it performs well on the letters that contains sharp edges.

\section{CONCLUSIONS}

Character recognition has served as one of the principal proving grounds for neural network methods and has emerged as one of the most successful applications of this technology. In our paper an on-line Arabic handwritten character recognition system based on Neural network is proposed. The experimental results shown that the machine has successfully recognized the characters string with the average 
accuracy of $83 \%$ which proof the effectiveness of our approach.

\section{REFERENCES}

[1] Vaseghi.B., Alirezaee.Sh., "Off-line Farsi/Arabic Handwritten word recognition using vectorquantization and hidden markov model," Proceedings of the 12th IEEE International Multitopic Conference, 978-1-4244$2824-3 / 08 / \$ 25.00$

[2] Dehghan M., Faez K., Ahmadi M.and Shridhar M., "Handwritten Farsi (Arabic) word recognition: a holistic approach using discrete HMM", Pattern Recognition, vol.34, no 5, pp. 1057-1065, 2001

[3] Chen M. Y., Kundu A., Srihari S. N., "Variable Duration Hidden Markov and Morphological Segmentation for Handwritten Word Recognition," IEEE Transactions on Image Processing, Vol. 4, No. 12, PP. 1675-1688, 1995.

[4] Kim G., Govindaraju V., "A Lexicon Driven Approach to Handwritten Word Recognition for Real-Time Applications," IEEE Transactions on Pattern Analysis and Machine Intelligence, Vol. 19, No. 4, PP. 366-379, 1997.

[5] Guillevic D., Suen C. Y., "HMM-KNN Word Recognition Engine for Bank Cheque Processing,"Proceedings of International Conference on Pattern Recognition, Vol. 2, PP. 1526-1529, Brisebane, Ausrtalia, August 1998.

[6] Adnan Amin,"Off-Line Arabic Character Recognition: The State of The Art", Pattern Recognition,Vol. 31, No. 5, 1998, pp. 517-530.

[7] Amin, A., Al-Sadoun, H., Fischer, S.,"Hand-printed Arabic character recognition system using an artificial network". Pattern Recognition, Vol. 29, No. 4, pp. 663 $675,1996$.

[8] Abdulkadr, A., "Two-tier approach for Arabic offline handwriting recognition". In: Proc. 10th Internet. Workshop on Frontiers in Handwriting Recognition (IWFHR), pp. 161-166.2006

[9] Karim Hadjar and Rolf Ingold, "Arabic Newspaper Page Segmentation", proceeding of the seventh international conference on document analysis and recognition, Vol. 2, 2003, pp. 895 - 899.

[10] T. S. El-Sheikh and S. G. El-Taweel, "Real-time Arabic handwritten character recognition", Pattern Recognition, Vol. 23, no.12, 1990, pp. 1323-1332.
[11] T. S. El-Sheikh and Ramez M. Guindi, "Computer recognition of Arabic cursive scripts", Pattern Recognition, Vol. 21, no. 4, 1988, pp. 293-302.

[12] F. El-Khaly and M. A. Sid-Ahmed, "Machine recognition of optically captured machine printed Arabic text", Pattern Recognition, Vol. 23, no. 11, 1990, pp. 12071214

[13] Mohamed S. El-Wakil and Amin A. Shoukry, "On-line recognition of handwritten isolated arabic char-acters", Pattern Recognition, Vol. 22, no. 2, 1989, pp. 97-105.

[14] Sabri A. Mahmoud, "Arabic character recognition using Fourier descriptors and character contour en-coding", Pattern Recognition, Vol. 27, no. 6, 1994, pp. 815-824.

[15] A. Cheung, M. Bennamoun, and N.W. Bergmann, "An Arabic optical character recognition system using recognition-based segmentation ", Pattern Recognition, Vol. 34, 2001, pp. 215 - 233.

[16] M.S. Khorsheed, "Recognizing handwritten Arabic manuscripts using a single hidden Markov model", Pattern Recognition Letters, Vol. 24, 2003, pp. 22352242.

[17] Neila Mezghani, Mohamed Cheriet, and Amar Mitiche, "Combination of Pruned Kohonen Maps for On-line Arabic Characters Recognition ", In proceedings of the Seventh International Conference on Document Analysis and Recognition (ICDAR 2003), pp. 900 - 904.

[18] J.H. AlKhateeb, J.Ren, J.Jiang, H.Al-Muhtaseb," Offline handwritten Arabic cursive text recognition using Hidden Markov Models and re-ranking", Pattern Recognition Letters 32 (2011) 1081-1088.

[19] B.Vaseghi, S.Hashemi," Farsi/arabic Handwritten Word Recognition Using Discrete HMM and Self-Organizing Feature Map", International Congress on Informatics, Environment, Energy and Applications-IEEA 2012 IPCSIT vol.38 (2012)

[20] A.T.Al-Taani, S.Al-Haj," Recognition of On-line Arabic Handwritten Characters Using Structural Features", Journal of Pattern Recognition Research 1 (2010) 23-37.

[21] M. Meyer, G. Pfeiffer, "Multilayer perceptron based decision feedback equalizers for channels with intersymbol interference,". IEEE Transactions,Vol 140, No 6, pp 420-424, Dec 1993.

[22] M. Ibnkahla, Signal Processing for Mobil Communications Handbook, Ed. Boca Raton, FL: CRC Press, 2005 